\title{
A física na visão de Ernst Mach: De uma crítica a Newton às teorias gravitacionais
}

\author{
The physics in the view of Ernst Mach: From a criticism to Newton to the gravitational theories

$$
\text { G. B. de Gracia*1] }
$$ \\ ${ }^{1}$ Universidade Estadual Paulista, Instituto de Física Teórica, São Paulo, SP, Brasil
}

Recebido em 29 de Novembro, 2018. Revisado em 14 de Janeiro, 2019. Aceito em 30 de Janeiro, 2019.

\begin{abstract}
O presente trabalho possui como objetivo introduzir conceitos chave da filosofia da ciência do físico austríaco Ernst Mach, assim como analisar a sua influência sobre teorias gravitacionais. Para tal, mostraremos que ela culmina numa crítica à ideia de espaço absoluto de Newton. Essa crítica teve considerável influência sobre Albert Einstein, entusiasta da filosofia de Mach, e o motivou a construir uma teoria que englobasse boa parte dos seus conceitos. Finalmente, analisaremos dois dos trabalhos originais de Einstein a fim de analisar a compatibilidade entre sua teoria gravitacional e as ideias de E.Mach . Assim, também comentaremos a respeito de uma generalização de sua teoria que visava incorporar toda a epistemologia Machiana.
\end{abstract}

Palavras-chave: Filosofia da ciência, gravitação, ideias de Mach e Einstein.

\begin{abstract}
The goal of the present article is to introduce the key concepts of the Ernst Mach's philosophy of science as well as to analyze its influence on gravitational theories. In order to do so, we show that it culminates in a criticism about Newton's absolute space idea. The mentioned criticism had considerable influence on Albert Einstein, an enthusiastic follower of Mach's ideas, such that it motivates him to create a theory that encompass great part of its concepts. Finally, we study the Einstein's originals papers in order to analyse the compabitility between his gravitational theory and the E.Mach's ideas. So, we also comment about one generalization that intend to incorporate all the Machian epistemology.
\end{abstract}

Keywords: Philosophy of science, gravitation, Mach's and Einstein's ideas.

\section{Introdução}

O físico e filósofo austríaco Ernst Mach, que viveu entre 1838 e 1916 e lecionou na universidade de Viena soube unir os seus conhecimentos e sua intuição física com as correntes filósoficas que influenciavam o pensamento de sua época. Ele possuía uma visão epistemológica fortemente influenciada pelo positivismo, movimento com desdobramentos filosóficos e sociológicos que surgiu na França no começo do século $X I X$, tendo como idealizadores Auguste Comte e Stuart Mill [1]. Essa escola sintetizou o "modus operandi"sócio-cultural do século mencionado e manteve-se muito influente até meados do século $X X$. Como exemplo de abordagem positivista, no que tange à ciência, pode-se citar a estrutura ultra pragmática, fenomenológica e anti-metafísica empregada na interpretação de copenhague da mecânica quântica, a qual é a mais aceita nos dias atuais [2].

A filosofia da ciência Machiana, em grande parte baseada no seu livro a Ciência da Mecânica [3], se destaca por uma abordagem fortemente fenomenológica. Isso significa que, no que se refere aos fundamentos da física, ele propunha uma mecânica na qual não fosse usada nenhuma noção abstrata de apoio que não pudesse ser posta à prova

*Endereço de correspondência: gb9950@gmail.com dos experimentos. A ideia era fundamentar a física com o menor emprego de noções metafísicas possível; devia-se apelar apenas para os dados experimentais e conceitos que de algum modo fossem acessíveis ao ser humano. $\mathrm{O}$ papel do filósofo da ciência seria o de analisá-los com o devido cuidado e criar uma estrutura para a física que não necessite apelar a conceitos inobserváveis. Tal tarefa não é nada trivial, pois muitas vezes nós o fazemos de uma maneira indireta. Assim, na presente discussão, empregaremos o termo inobservável quase como sinônimo de metafísico. Um conceito desse tipo é o do éter. Embora ele tenha sido empregado como uma noção de apoio, um modelo essencial para a construção do eletromagnetismo de Maxwell [4], nunca poderemos refutá-lo, haja vista que este conceito é construído de tal modo que até a falha em detectá-lo, no experimento de Michelson-Morley, pode ser naturalmente contornada com o argumento do arrasto do éter. Logo, ao menos sob a ótica de Karl Popper 5] (que também possuia influências positivistas), o éter seria uma noção não científica. Embora com o uso deste conceito tenha sido possível prever resultados experimentais, Mach e os demais fenomenologistas optaram por considerar estes "conceitos de apoio"como apenas modelos subjetivos usados para se extrair observáveis.

A presente exposição tratará da abordagem Machiana 
(contida no livro, A Ciência da Mecânica.) dos fundamentos da teoria Newtoniana [6], a qual tem suas origens remotas, ainda no século $X V I I$, com as críticas de Leibniz e Berkeley 7]. Dessa maneira, apresentaremos a crítica de Mach ao conceito de espaço absoluto em oposição à noção de um espaço puramente referencial, analisaremos teorias cosmológicas sob o seu ponto de vista, e o seu conceito relativista de massa inercial e gravitacional. Comentaremos a respeito da compatibilidade entre a relatividade de Einstein e a perspectiva Machiana e, finalmente, sobre a teoria gravitacional de Brans-Dicke [8] a qual visa incorporar integralmente os conceitos Machianos, algo que, como mostraremos, não ocorre na teoria de Einstein .

\section{A experiência do balde}

Logo após a publicação dos Principia Mathematica Philosophiae Naturalis 9], no final do século XVII, e o seu grande sucesso em explicar a mecânica celeste do sistema solar, Newton foi alvo de especulações filosóficas a respeito dos fundamentos de sua teoria da mecânica. Filósofos como Leibniz, homem de grande cultura que atuou em diversas áreas, desde o direito até a matemática, e o bispo Berkeley, com importante contribuição na filosofia, não encontravam uma motivação teórica para um espaço absoluto, conceito, esse, no qual Newton se baseou.

De acordo com esses senhores, não há razão para se supor propriedades ao espaço que não sejam puramente referenciais. Ou seja, não faria sentido empregar a noção espaço na presença de um único corpo, mas apenas com relação a algum sistema de referência. Assim, a realidade física deveria ser sempre descrita por grandezas que relacionem um sistema a outro, sem a necessidade de postular um espaço absoluto com significado intrínseco. Ao elevar isso como princípio, Leibniz afirmou que tanto a teoria Ptolomaica, que punha a terra no centro do sistema solar, quanto a teoria heliocêntrica, eram igualmente válidas sendo esta última uma abordagem mais clara e simplificada da situação física. Uma teoria construída nesses moldes, no século $X I X$, foi a chamada eletrodinâmica de Weber a qual utiliza apenas grandezas relacionais 7 ]. No século $X V I I I$, o filósofo I. Kant, em seu livro Crítica da razão pura [10], argumenta que o espaço e o tempo são conceitos que o ser humano necessita de antemão para fixar o seu entendimento do mundo, mais especificamente, para intuir a sucessão e a simultaneidade. O que se pode depreender disso é a natureza meramente relacional do espaço e do tempo. Além disso, a concepção Kantiana de espaço e tempo os coloca num status muito parecido analogamente ao que viria a ser realizado na teoria da relatividade Einsteniana.

Newton, contemporâneo dos dois filósofos citados no início desta seção, precisou dar uma resposta às suas indagações a fim de fundamentar epistemologicamente a ideia de espaço absoluto que vinha empregando. Para isso, ele propôs uma experiência [7], [11] conhecida como a do balde de Newton: Se mantivermos um balde com água em repouso em relação à Terra, a superfície da água estará plana. A experiência mostra que, se o balde for posto a girar em relação a Terra, então, gradativamente, a água também entrará em rotação e passará a se afastar do meio do recipiente e subir pelos seus lados, assumindo uma forma côncava (ação de uma aparente força centrífuga). De acordo com Newton, esse efeito só é possível devido à rotação da água em relação ao espaço absoluto, e não em relação ao balde, à Terra ou às estrelas fixas. Dessa maneira, se o balde com água estivesse em repouso em relação ao espaço absoluto e todo o restante do universo estivesse em rotação em relação a ele, a superfície da água continuaria plana. Newton conclui então que a formação da concavidade na água do balde é o dado experimental que nos permite distinguir entre um movimento relativo qualquer e aquele feito com relação ao espaço absoluto.

A menção às estrelas fixas provavelmente se deu devido ao fato de que os defensores de uma ciência referencial supunham que o primeiro passo para refundar a teoria Newtoniana era substituir o espaço absoluto por um sistema de referência relativo às estrelas fixas, aquelas que estão muito distantes da terra e como a distância delas é muito grande em comparação às dimensões do sistema solar, elas configurariam um referencial praticamente inercial.

O argumento desenvolvido por Newton foi usado a fim de derrubar a concepção relacional do espaço. Esse argumento resistiu durante muito tempo e ao menos Leibniz e Berkeley não conseguiram mais se opor eficientemente a ele. O sucesso experimental da teoria da gravitação universal também serviu para calar eventuais críticos.

$\mathrm{O}$ que Mach trouxe de novo à discussão foi uma atitude radical à respeito da interpretação da experiência do balde. Se não há motivações a priori para o espaço absoluto e se apenas podemos experienciar o espaço dado com relação aos demais corpos, deve existir sempre uma influência mutuamente simétrica entre dois sistemas físicos. Tal princípio o levou a supor que se o próprio sistema de estrelas fixas (usado a fim de fixar um referencial inercial adequado) fosse girado com relação ao balde, a situação física deveria ser simétrica e, portanto, a água do balde deveria tomar uma forma abaulada. Assim, foi possível ao menos dar uma resposta ao argumento de Newton. Uma conclusão que se pode tirar desta sua observação é a de que o movimento relativo entre diferentes observadores pode gerar efeitos físicos. Além disso, a física local deve levar em conta a distribuição de massas no universo, o que numa perspectiva moderna seria a de massa-energia.

A relatividade geral já leva em conta alguns aspectos das ideias de Mach, como a influência da distribuição de matéria-energia em fenômenos físicos e a criação de uma física independente do referencial adotado. 


\section{O colapso da cosmologia Newtoniana}

A teoria de Newton obteve sucesso em explicar o movimento dos corpos celestes no sistema solar. Tratava-se de uma teoria que descrevia desde fenômenos que ocorriam na órbita da lua em torno da terra até outros que ocorriam em regiões distantes do sistema solar, o que justificou a nomenclatura de gravitação universal. Este triunfo levou os teóricos a extrapolarem qualquer limite observacional e a usarem a gravitação universal como meio de se analisar toda a cosmologia. Um olhar mais pragmático e atento deveria levar em consideração que esta teoria foi construída por meio de princípios propostos por Galileu Galilei e das três leis de Kepler [12]. Devido a esse fato, Newton pôde embasar sua teoria em dados experimentais muito confiáveis de Tycho Brahe, nos quais as leis de Kepler se basearam.

Não se pode ignorar o fato de que tais dados se referiam basicamente a movimentos de planetas e demais corpos no sistema solar. Isso significa que estender a teoria Newtoniana para todo o universo seria um grande salto, pois ela se baseou em leis e dados experimentais que diziam respeito apenas ao sistema solar.

Quanto à elevação de sua teoria ao status de cosmologia, Newton inicialmente acreditava que o espaço se estendia infinitamente porém a distribuição de matéria se dava numa região de volume finito. Podemos notar aí uma grande divergência do que viriam a ser as ideias de Mach e também de contemporâneos de Newton, como Leibniz, em que o espaço vazio de matéria, ou qualquer tipo de referência simplesmente não faria sentido, pois perderia seu aspecto relacional e seria então puramente metafísico. Ao fazer menção às estrelas fixas, que por estarem muito distantes funcionam como uma boa aproximação para um referencial inercial, Newton conjecturou que elas, assim como o sol, poderiam ser o centro de sistemas análogos ao solar. Para se fundar uma teoria consistente era preciso evitar que as estrelas fixas se atraíssem gravitacionalmente levando a um colapso da sua cosmologia, o que pelo ponto de vista de Mach seria não só o colapso da cosmologia quanto do próprio significado de espaço, haja vista que o colapso da matéria num ponto tornaria impossível a intuição do espaço pois se estaria na ausência de outros sistemas que poderiam servir como referência.

Para contornar essa situação, Newton supôs que as estrelas fixas estavam muito distantes umas das outras. Essa solução não resolve o problema, pois o potencial gravitacional do tipo $1 / r$ tem extensão infinita e, por isso, num dado instante o sistema colapsaria. Então, ele passou a assumir que o conteúdo material do universo estaria espalhado por todo o volume infinito do espaço, conforme pode-se verificar numa carta que ele enviou ao teólogo Bentley ( 13 ; pág. 281) . Nota-se que acordo com Mach, apenas dessa forma se poderia pensar um espaço infinito, mas para Newton o espaço possuía um significado absoluto. A fim de evitar o mencionado co- lapso, Newton supôs que a matéria estaria distribuída num espaço infinito de maneira uniforme [7]. Ele acreditava que com isso resolveria a questão, mas na realidade o colapso seria evitado apenas num dado referencial.

Ao usar a teoria Newtoniana, concluímos que ao por um corpo massivo na origem de nosso sistema de coordenadas supondo um universo infinito com uma quantidade de matéria infinita distribuída uniformemente, como pretendia Newton, o problema seria idêntico ao de uma massa circundada por um conjunto infinito de cascas esféricas e a resultante sobre a partícula seria nula, levando a uma situação de equilíbrio. Agora, se analisarmos a mesma situação com a origem transladada na direção de um dado vetor, obteremos uma força resultante na direção deste vetor. Este foi um paradoxo para o qual apenas anos mais tarde surgiram respostas satisfatórias.

Ao analisar esta situação sob a visão Machiana concluise que antes de mais nada deve-se tomar um bom referencial inercial o qual, no caso, seria o das estrelas fixas. Os dois sistemas de referência citados anteriormente, dados com relação ao das estrelas fixas são igualmente bons e não haveria como surgir forças de inércia apenas devido a sua troca. Como não é isso que se verifica, notamos que ao extrapolar a física de Newton à nível cosmológico, acabamos por ter uma nítida percepção de sua suposição de espaço absoluto, evidenciada no caso desse paradoxo, pois tem-se a impressão de que a referida massa, para não sofrer uma força resultante, deveria estar no centro do espaço absoluto. Ou seja, a teoria de Newton foi construída sobre este conceito e acaba por implicar nele.

Uma solução para este problema está em supor uma modificação do potencial gravitacional, proposta por C. Neumann [7], para um do tipo $V(r)=-\frac{G M e^{-\lambda r}}{r}$, no qual o parâmetro $\lambda$ controlaria o comprimento de correlação do sistema. Como a teoria funciona bem no sistema solar supõe-se que tal parâmetro seja tal que só tenha uma influência apreciável em distâncias muito maiores que a do referido sistema. Ao utilizar esse potencial potencial modificado evita-se o colapso da cosmologia de Newton. Como veremos, a inclusão de um termo de constante cosmológica nas equações de Einstein visava resolver esse mesmo problema.

\section{A visão Machiana sobre a determinação das massas inerciais e gravitacionais}

Sabe-se que Newton já levava em consideração que poderia haver uma diferença, ao menos conceitual, entre a massa inercial que é aquela que representa a proporcionalidade entre a força aplicada num corpo e a aceleração que ele adquire, e a massa gravitacional que numa visão pragmática é a carga da gravitação. Inspirado pela obra de Galileu ele supôs a igualdade destas, ao menos para corpos pontuais ou esféricos. Quanto a sua definição ele afirmou que "a quantidade de matéria é a medida da 
mesma, se originando de sua densidade e volume" 9 . Veremos que Mach nos proverá com uma definição mais elaborada, mais vinculada ao conceito relacional.

De acordo com as idéias de Mach, as forças possuem um caráter intrinsecamente ligado aos referenciais utilizados, uma vez que as grandezas físicas devem depender simetricamente apenas de quantidades que envolvam as de dois ou mais sistemas que servem de referência mútua uns aos outros.

Assim, ao tomar o referencial das estrelas fixas, que para experimentos que ocorrem na superfície da terra é mais aproximadamente inercial do que a própria terra, e ao avaliar o resultado da colisão de dois corpos com relação a ele, de acordo com a lei de ação e reação, surgirão forças iguais e opostas agindo neles o que nos permite fixar que a razão de suas massas inerciais é o inverso da razão de suas acelerações com relação ao referido referencial. Ao tomar uma massa como padrão, por exemplo $1 \mathrm{~kg}$, define-se a massa do outro corpo. Desse modo, o cálculo da massa é relativo a um dado sistema de coordenadas e a um outro corpo de teste. O espaço absoluto e a massa absoluta perdem seu sentido. De certa forma, isso foi incorporado na relatividade restrita ao se considerar a dependência da massa com a velocidade do referencial inercial. Curiosamente, é interessante citar que o eletromagnetismo, que deve grande parte de seus resultados ao modelo subjetivo do éter, também obteve conclusões similares relacionados a dependência da massa com a velocidade [4].

Quanto à massa gravitacional, Mach considera tomarmos como referência as estrelas fixas [7, analisando a atração gravitacional de dois corpos, sendo que se fixa a massa inercial de um deles como o padrão de unidade $1 \mathrm{~kg}$ e avalia-se a aceleração que este corpo recebe numa distância infinita (na realidade muito grande.) entre eles. Esse cuidado deve ser tomado para evitar efeitos multipolares decorrentes da distribuição de massas dos corpos 14.

$$
m_{g}=\lim _{r \rightarrow \infty} \frac{m_{I} a r^{2}}{1 k g \times G}
$$

$\mathrm{Na}$ expressão acima figura a massa inercial que se supõe conhecida através do procedimento experimental descrito anteriormente. A variável $a$ designa a aceleração experimentada pelo corpo com relação ao referencial adotado.

\section{A teoria de Einstein e os conceitos Machianos}

Esta seção será baseada no artigo de Einstein de 1916 15] que introduz a relatividade geral e terá como objetivo analisar sua compatibilidade com os conceitos Machianos. Assim, primeiramente, analisaremos a sua fundamentação conceitual.

\subsection{Fundamentação Epistemológica}

A teoria da relatividade geral de Einstein, visava generalizar o princípio da relatividade restrita, o qual se referia apenas a uma classe de referenciais, os inerciais. Vale a pena salientar que esta teoria já incorporava um certo viés Machiano devido a insistência num caráter relativista, mesmo que parcial, e na substituição do conceito do éter por um dado experimental [4] . Para esses referenciais a física seria a mesma. Nesta teoria estamos restritos ao uso de coordenadas holônomas, pois a admissão de coordenadas curvilíneas levaria a efeitos interpretados como forças inerciais.

A diferença entre a relatividade especial e a mecânica clássica está no fato de que a primeira concilia o eletromagnetismo com uma determinada classe de referenciais privilegiados para os quais a física é a mesma. Houve apenas uma extensão do princípio de Galileu. Apesar disso, não podemos deixar de verificar importantes diferenças oriundas dessa extensão. Por exemplo, foi fundamental assumir que a velocidade da luz no vácuo é constante, além disso, o espaço-tempo de Newton que era do tipo $\mathcal{R} \times \mathcal{R}^{3}$, com o tempo assumindo um status diferente daquele da parte espacial, (no qual as simetrias espaçotemporais geram translações no tempo, representado pela reta real, e rotações e translações no espaço tridimensional), é substituído pelo espaço chamado de Minkowsk ${ }^{1}$

$\mathrm{Na}$ relatividade especial, a estrutura das transformações de Lorentz, a quantidade invariante que é o intervalo, sugerem agora um espaço tempo de natureza hiperbólica, pseudo-Euclidiano e quadridimensional do tipo $\mathcal{R}^{4}$. Nesse espaço o tempo assume o mesmo status de uma coordenada espacial. Essa diferença possui consequências na estrutura causal do espaço em questão; enquanto que no espaço-tempo de Newton, quaisquer eventos podem ser casualmente conectados, na relatividade especial, devido à finitude da velocidade das interações, há três classes de intervalos entre eventos, os do tipo tempo, do tipo luz e do tipo espaço, sendo que apenas os dois primeiros podem ser casualmente conectados. Também podemos citar o conceito da relatividade da simultaneidade, dilatação temporal e contração espacial. Essa extensão da classe de referenciais inerciais também trouxe novidades aos conceitos de matéria e energia e momento, relacionandoos através da equação $E^{2}=m^{2} c^{4}+\vec{p}^{2} c^{2}$.

Apesar destes avanços, é claro que havia a necessidade de se generalizar tal teoria, haja vista que ela não leva em conta referenciais com movimento relativo não-uniforme de modo que para esses referenciais, a física decorre de modo diferente, surgindo forças de inércia. Novamente, lembramos que do ponto de vista relacional, ou seja, partindo do princípio com respaldo fenomenológico de que todos os eventos a que temos acesso se dão de modo que o espaço-tempo é uma entidade meramente de referência, algo usado apenas para relacionar eventos, que não

${ }^{1}$ Espaço cujo grupo de isometria é definido pela invariância do intervalo definido por $d s^{2}=-d \vec{x}^{2}+c^{2} d t^{2}$, trata-se uma estrutura que lida com o espaço e o tempo de maneira unificada. 
possui significado intrínseco, a menos que se faça considerações metafísicas. Porém essas seriam considerações não-mínimas, e se nos atermos apenas à realidade física a que temos acesso, o princípio relacional parece o mais adequado.

Um evento que relaciona dois sistemas poderia a princípio admitir qualquer parametrização caso seja possível criarmos um mapa inversível entre esta descrição e uma outra qualquer. Ambas deveriam ser equivalentes pois suas funções são apenas as de descrever relações entre os mesmos eventos por meio de diferentes coordenadas. Esse princípio nos leva a considerar que a correta descrição da realidade física deve se dar através de uma teoria invariante por transformações gerais de coordenadas (TGC).

Assim, Einstein faz uso de considerações heurísticas a fim de demonstrar a necessidade de uma teoria mais geral para a relatividade. Ele considera dois sistemas $R_{1}$ e $R_{2}$ que giram em torno de uma linha imaginária transversal à linha que os une, sendo que em cada um desses sistemas estão os corpos deformáveis inicialmente esféricos 1 e 2, respectivamente. A situação é evidentemente simétrica, mas se um observador é colocado em $R_{1}$, ele nota que o corpo 2 se move de modo não uniforme com relação a ele e que devido ao surgimento da força de inércia, no caso a centrípeta, o segundo corpo passa a se deformar, adquirindo a forma de um elipsóide. Esse é um resultado observável, corresponde a uma realidade física. A primeira vista esta consideração parece violar a noção de espaço referencial, fortemente defendido por E.Mach, filósofo da ciência que influenciou muito as ideias de Einstein, pois a física em $R_{1}$, em relação ao qual o corpo 1 está em repouso, é diferente da de $R_{2}$. Seria preciso derivar essa força como algo oriundo do próprio princípio da relatividade ( $\mathrm{O}$ espaço tempo não é intuído de modo absoluto, mas sim a depender do sistema de referência.), pois assim esta força estaria incorporada ao ferramental teórico da mecânica, o que permitiria tratar qualquer referencial em status de igualdade. Assim, a física de fato seria a mesma para todos eles.

Para responder a esta questão Einstein propôs outra reflexão. Supõe dois sistemas $K$ e $K^{\prime}$ que estão se separando por uma aceleração constante. Como o movimento é relativo, um observador em repouso em $K^{\prime}$ não poderia deduzir o seu estado de movimento, ou seja, perceber-se acelerado, pois essa aceleração é um conceito relativo entre dois sistemas e não algo intrínseco ao mencionado referencial. Para esse observador é $K$ que se move de modo acelerado.

Partiremos do princípio de que as massas gravitacionais e inerciais são as mesmas (princípio de equivalência fraco [16]), o que possui verificação experimental dada por Eötvos e nos dias atuais por [17]. Sendo assim, numa região infinitamente próxima à $K^{\prime}$, onde não se pode medir gradientes da aceleração gravitacional [14], a fenomenologia observada neste sistema com relação ao movimento de $K$ pode ser entendida como oriunda de um campo gravitacional, ou seja a aceleração observada pode ser entendida como resultante de uma interação gravitacional (Princípio de equivalência forte [18]). Desse modo, as forças de inércia deixam de ser um sinal da não equivalência física entre sistemas de referência e passam a ser incorporadas dentro do esquema relativista, passando a ser entendidas como manifestação da gravitação, o que eleva todos os referenciais ao mesmo status.

Vale a pena observar que esta condição mencionada acima deve ser entendida de modo mais abrangente se levarmos em conta que no caso em que há uma densidade de férmions no espaço tempo, a torção, inicialmente considerada nula, passa a ser proporcional a densidade de spin naquela região. Isso afeta as equações de Einstein e, portanto, o campo gravitacional.

Assim, a aceleração da gravidade não estaria relacionada apenas a um efeito de referencial e sim, em parte, a uma propriedade intrínseca de uma determinada região do espaço-tempo. Mas se admitirmos que a região considerada é muito pequena, a densidade de spin é a mesma para dois referenciais contidos nela e suas diferenças dinâmicas se deveriam novamente a efeitos de referencial. Esta seria a conjectura de uma generalização do princípio de equivalência. Estas questões estão relacionadas à abordagem de Einstein-Cartan da relatividade geral e são didaticamente introduzidas em [19]. Isso nos mostra que a teoria de Einstein tem uma boa fundamentação macroscópica, porém em nível microscópico questões não triviais podem surgir.

As observações anteriores nos levam à conclusão de que o princípio da relatividade demandaria uma física invariante por TGC cujas forças de inércia são elevadas ao status de interação gravitacional, sendo que veremos que ela está codificada no desvio da geometria atual com relação à Minkowskiana. A necessidade de se abandonar a métrica de Minkowski num caso geral é devido ao fato de que ao considerar sistemas animados de movimento relativo não uniforme pode-se inferir que o espaço-tempo, do ponto de vista deles, deixa de ser plano e Minkowskiano e passa a ser curvo. Então, uma consideração importante é a de que é preciso um objeto tensorial $R_{\mu \nu \rho \sigma}$ construído apenas com derivadas da função métrica (que define o produto escalar generalizado representado pelo intervalo $d s^{2}$ ) e que portanto se anula caso ela for constante (ou seja, numa região com geometria plana). Como um tensor nulo o é em todos os sistemas de referência, caso dispormos de uma métrica com dependência espaço-temporal, mas que é gerada por meio de uma TGC agindo sobre a de Minkowski, a nulidade do tensor $R_{\mu \nu \rho \sigma}$ acusaria o fato de que não há campo gravitacional real e que o desvio com relação ao espaço plano se dá de modo artificial, meramente um efeito de se descrevê-lo por meio de um sistema de coordenadas mais complicado. Este é o tensor de Riemann-Christofell. Por enquanto, inferiremos a mencionada necessidade de se abandonar, num caso geral, a geometria pseudo-euclidiana de Minkowski.

Tal conclusão foi obtida por Einstein por meio de um experimento mental. Ele considerou dois observadores, 
sendo que um estava na origem de um dado sistema de coordenadas e o outro fazia um movimento circular uniforme em torno dessa origem. Ao aplicar conceitos da relatividade restrita conclui-se que aquele observador que gira, devido à sua velocidade tangencial à circunferência que ele perfaz, nota um perímetro maior que aquele que seria medido pelo observador em repouso na origem pois este vê o espaço contraído. Como sua velocidade é ortogonal ao raio da esfera este não varia. Logo, o observador na origem conclui que a razão entre esse perímetro e o raio vale $\pi$, enquanto que aquele animado de velocidade angular conclui que esta razão é maior que $\pi$, pois ambos concordam com relação ao raio, mas aquele que está em repouso nota um perímetro menor que aquele verificado pelo primeiro. Portanto, é preciso assumir geometrias mais abrangentes que englobem espaços que podem possuir uma curvatura intrínseca. Ao admitir sistemas animados de todos os tipos de movimento relativo, deve-se abandonar também a ideia da constância da velocidade da luz, pois se para um observador ela se propaga em linha reta, para outro ela se move de maneira curva, modificando sua velocidade. Essa possibilidade dá margem a fenômenos como os das lentes gravitacionais que nada mais são que fenômenos de desvio gravitacional da luz.

\subsection{A cosmologia de Einstein e as idéias de Ernst Mach}

Com a intenção de erigir uma teoria cosmológica [20], Einstein analisa primeiramente a cosmologia de Newton. Dessa maneira, ele comenta que além da equação de Poisson ${ }^{2} \nabla^{2} \phi=4 \pi G \rho$ para o potencial gravitacional $\phi(x)$, uma estrutura local para a gravitação universal necessitaria de considerações sobre o comportamento do potencial no infinito espacial. Na relatividade geral a situação é análoga, se conjecturarmos um universo espacialmente infinito, precisamos conhecer qual forma a métrica ${ }^{3} g_{\mu \nu}(x)$ assume para grandes distâncias. Ao analisar o movimento de planetas no sistema solar considerou-se que a métrica tendia a um valor constante para uma região muito distante dessa distribuição de massa. Mas para extensões maiores do mundo astral, seria legítimo assumir estas mesmas condições, ou elas levariam a ocorrência de alguma situação cosmológica absurda? Como não há razão a priori para se assumir essas condições

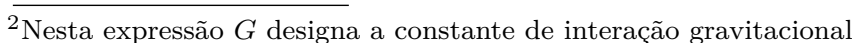
e $\rho(x)$ a densidade local de matéria.

${ }^{3} \mathrm{~A}$ teoria da relatividade geral prevê a necessidade de se adotar um espaço tempo não-Euclidiano como se infere dos experimentos mentais de Einstein. Assim o elemento de linha generalizado passa a ser dado pelo teorema de Pitágoras quadridimensional generalizado $d s^{2}=g_{\mu \nu}(x) d x^{\mu} d x^{\nu}$ com o quadrivetor $d x^{\mu}=(c d t, d \vec{x}), g_{\mu \nu}(x)$ é chamada de métrica do espaço tempo e sua forma depende da distribuição local de matéria. Ou seja, de acordo com Einstein, a matéria curva o espaço-tempo. Estamos considerando a convenção de Einsten na qual índices repetidos acima e abaixo estão sendo somados, e $\mu=1, \ldots, 4$ sendo que 4 designa a componente temporal dos quadrivetores.
}

Einstein se propôs fazer algumas reflexões que serão abordadas nas próximas seções.

\subsubsection{A análise da cosmologia de Newton}

Em geral, nas aplicações da gravitação Newtoniana assume-se um potencial gravitacional constante no infinito espacial. Assim, tendo em vista a equação de Poisson e o fato de que uma derivada espacial ao menos dimensionalmente é algo do tipo $\nabla^{2} \sim \frac{1}{r^{2}}$ para que

$\phi(r \rightarrow \infty) \sim \lim _{r \rightarrow \infty} 4 \pi G \rho r^{2}$ tenda a um valor constante é necessário que a densidade de matéria se comporte como $\rho(r \rightarrow \infty) \sim\left(\frac{1}{r^{2}}\right)^{\gamma} \operatorname{com} \gamma \geq 1$. Assim, a densidade de matéria se desvanece até se tornar assintóticamente nula para grandes distâncias. É preciso ter em mente que $\rho$ nas presentes considerações representa a densidade média de matéria para escalas muito maiores que as distâncias interplanetárias mas muito menores que as dimensões do universo. Essas condições de contorno implicam que o universo possui um centro absoluto, de densidade máxima, a partir do qual a densidade de matéria passa a diminuir. Esta característica é problemática do ponto de vista relativista e nos chama a atenção para o fato de que para baixas velocidades a relatividade geral tende à teoria Newtoniana e portanto passaria a violar seu próprio princípio fundamental. Chega-se a conjecturar modificações da equação de Poisson. Um comentário interessante é que tal universo é espacialmente finito, pois se o espaço é puramente relacional e como a matéria está distribuída numa região finita, ali deve se delimitar o universo, pois o espaço totalmente vazio é uma entidade puramente metafísica, da qual não se pode extrair nenhuma informação física.

Logo, se este universo é finito, o potencial $\phi$ assume um dado valor constante em sua fronteira e, a princípio, um planeta de massa $m_{p}$ animado de uma energia cinética suficiente para superar a barreira de potencial $m_{p} \phi$, escaparia da região onde se definiria tal universo. Ao levar em conta a física estatística e, portanto, a possibilidade de flutuações na distribuição de velocidades dos corpos celestes, esta situação ocorreria fatalmente com uma dada frequência e ocorreria o desvanecimento gradual da matéria do universo. Para evitar tal situação se poderia pensar em impor um valor muito alto para esse potencial no extremo do universo. Mas deve-se prestar atenção no fato de que haveria assim uma grande diferença de potencial entre o centro do universo e sua fronteira, o que condicionaria os corpos celestes a apresentarem grandes velocidades, fato que não se observa experimentalmente.

Agora, se considerarmos o universo como um gás no qual os corpos celestes fazem o papel das suas moléculas e aplicarmos a lei de Boltzmann [21] para elas, chegaríamos numa situação de absurdo. A densidade de um gás é condicionada pelo potencial de interação entre suas "moléculas"e pela temperatura $\rho=\rho_{0} e^{-\frac{\phi}{K T}}$. Como entre o centro absoluto do universo e sua fronteira há uma 
diferença de potencial finita, a razão entre as densidades nestas duas regiões é finita e dada pela expressão:

$$
\frac{\rho_{c}}{\rho_{f}}=e^{-\frac{\phi_{c}-\phi_{f}}{K T}}
$$

Em que K designa a constante de Boltzmann e $\phi(x)$ é o potencial gravitacional.

Como a densidade na fronteira tende a um valor nulo, para manter a razão finita é preciso que $\rho_{c}$ tenda a zero, o que contradiz toda a construção teórica deste modelo cosmológico.

Estas observações demonstram que a teoria de Newton necessita ser modificada para que sua cosmologia não sofra inconsistências. Para tal, postularemos a seguinte modificação da equação de Poisson:

$$
\nabla^{2} \phi-\lambda \phi=4 \pi G \rho
$$

A adição do novo termo $\lambda \phi$ à equação acima permite que para uma densidade média de matéria constante $\rho=\rho_{0}$ exista uma solução do tipo:

$$
\phi=-\frac{4 \pi G}{\lambda} \rho_{0}
$$

Novamente é preciso ter em mente que se trata de uma aproximação, em largas escalas $\rho \sim \rho_{0}$ porém, localmente, a distribuição $\rho$ é altamente não uniforme. Assim, próximo a um planeta, o potencial $\phi$ se assemelhará muito a um campo Newtoniano se $\lambda \phi<<4 \pi G \rho$, o que asseguraria que a gravitação universal de Newton, assim como as observações experimentais, seriam recuperadas localmente. Quanto à solução do potencial $\phi$ para largas escalas, ela implica na ausência de um centro do universo a partir do qual a densidade de matéria desvanece, o que agrada muito as ambições relativistas 4 , e também podemos inferir o fato de que há apenas pequenos gradientes locais do potencial, o que está de acordo com a pequena velocidade observada dos corpos celestes com relação à velocidade da luz. Além disso, como a densidade de matéria é constante nesse universo, a aplicação da distribuição de Boltzmann não leva à ocorrência de uma contradição.

\subsubsection{Condições nos limites para a relatividade geral}

No caso da relatividade geral, no limite em que a razão $\frac{v}{c}$ entre a velocidade da partícula e da luz $c$ tende a zero, é necessário que se recupere a equação de Poisson modificada a fim de que em nenhum limite imaginável a relatividade geral recupere uma teoria que tenha como

\footnotetext{
4 Caso supormos uma densidade de matéria constante e usarmos a equação de Poisson sem modificação, nota-se que o universo estará em equilíbrio apenas num dado referencial privilegiado sendo que nos demais haveria colapso 7 .
}

implicação um referencial com status privilegiado. Do ponto de vista fenomenológico, obviamente a motivação para tal modificação ja foi dada no fim da seção anterior. O que é interessante de se observar é que ao retomarmos a discussão sobre as condições do potencial no infinito espacial, elas simplesmente deixam de ter cabimento, haja vista que se supõe a homogeneidade da distribuição de matéria e do potencial. Assim, Einstein é levado a supor um universo espacialmente fechado para o qual ele acreditava que as condições assintóticas do potencial não seriam relevantes.

A fim de reforçar esta última ideia, Einstein lança mão de considerações epistemológicas a respeito da relatividade da inércia, conceito de caráter Machiano compatível com a fundação conceitual de sua teoria relativista. Basicamente trata-se do princípio de que para avaliarmos uma massa, é preciso de ao menos outro corpo a fim de colidi-los, sob um referencial inercial adequado e, assim inferir a razão entre tais massas por meio da razão inversa de suas acelerações. Isso significa que o conceito de massa depende do referencial e também da presença de demais massas a fim de gerar efeitos observáveis que nos permitam inferir sua existência.

A relatividade geral seria compatível com a possibilidade de que uma partícula perderia sua inércia ao ser isolada de todas as demais? É a esta questão que Einstein se põe a considerar e veremos que ela está ligada às condições assintóticas do potencial, assunto relevante de seu trabalho sobre cosmologia.

No contexto da relatividade geral, o momento espacial é dado por:

$$
p_{i}=m \sqrt{-g} g_{i \alpha} \frac{d x^{\alpha}}{d s}
$$

Em que $g$ designa o determinante da métrica e $m$ designa a massa de repouso.

Como nesta discussão tem-se em vista aplicações cosmológicas lança-se mão do fato experimental de que a velocidade dos corpos celestes é muito menor que a da luz no vácuo. Assim, $d s^{2}=g_{\mu \nu} d x^{\mu} d x^{\nu} \sim g_{44} d x_{4}^{2} \bigsqcup_{5}^{5}$ sendo que de modo geral, ao considerarmos isotropia espacial, temos:

$$
d s^{2}=-A\left(d x_{1}^{2}+d x_{2}^{2}+d x_{3}^{2}\right)+B d x_{4}^{2}
$$

Para fazer os cálculos é conveniente adotar a classe de referenciais com $\sqrt{-g}=1=\sqrt{A^{3} B}$. Agora, ao considerar novamente o limite $\frac{v}{c} \sim 0$ temos:

$$
p_{i}=-m \frac{A}{\sqrt{B}} \frac{d x_{i}}{d x_{4}} \quad ; \quad E=m \sqrt{B}
$$

Sendo que $E \equiv p_{4}$ designa a energia.

Ao tomar como princípio a relatividade da inércia, é preciso requerer que a massa efetiva se anule na ausência de demais massas de referência:

\footnotetext{
${ }_{5 \text { pois temos a definição } d x_{4}} \equiv c d t$
} 


$$
m_{e f .}(r \rightarrow \infty) \equiv m \frac{A}{\sqrt{B}} \sim 0 \rightarrow A(r \rightarrow \infty) \sim 0
$$

Para a classe de referenciais que escolhemos isso significa que $B(r \rightarrow \infty) \rightarrow \infty$ para que se mantenha $\sqrt{-g}=1$. Sob o ponto de vista cosmológico essa última característica parece ser bem vinda pois a energia $E$ se torna muito grande para distâncias espaciais consideráveis e por isso o universo não sofreria do fenômeno do despovoamento. Por outro lado, uma variação tão brusca na energia potencial condicionaria os corpos celestes a apresentarem altas velocidades, fato que não se observa.

Ainda intrigado a respeito das condições assintóticas do potencial gravitacional Einstein e um matemático, J. Grommer, investigaram a existência de campos estáticos, com centro de simetria e que desvanecem no infinito espacial. Com tal métrica, calculou-se o tensor de energia-momento que seria sua fonte e verificou-se que ele não era compatível com o sistema de estrelas fixas, como salientou De-Sitter. Para entendermos isso, analisamos $T^{\mu \nu}=\rho \frac{d x^{\mu}}{d s} \frac{d x^{\nu}}{d s}$ que é o tensor de energia momentd 6 associado a uma distribuição de massas sem gradientes o que leva à ausência de pressões para manter o equilíbrio. Tais escolhas correspondem ao que Einstein conjecturou ao postular a modificação da equação de Poisson no contexto da cosmologia de Newton. Como a velocidade das estrelas é muito menor que a da luz, pode-se adotar um sistema de coordenadas no qual apenas $T^{44}=\rho \neq 0$. Como já mencionado, esta condição não é compatível com o desvanecimento da parte espacial de $g_{\mu \nu}$ no infinito espacial e portanto parece não ser compatível com a relatividade da inércia. É necessário resolver o conflito entre a existência de uma característica puramente relacional para a matéria e a existência de um universo estável e consistente logicamente.

Como conciliar estes princípios? Poderia-se supor que para $r \rightarrow \infty$ há sempre um sistema de coordenadas para o qual $g_{\mu \nu}(r \rightarrow \infty) \rightarrow \eta_{\mu \nu}$. Mas ao analisarmos a inércia efetiva $m \frac{A}{\sqrt{B}}$, conclui-se que ela é influenciada pela distribuição de massas no universo, mas como $g_{\mu \nu}$ difere pouco de $\eta_{\mu \nu}$, a existência ou não de demais massas no universo não condicionaria a existência de sua inércia; uma partícula, nesse caso, teria inércia mesmo se estivesse solitária no universo.

Além disso, devido a variação de potencial, existiria o desvanecimento da densidade de matéria no infinito espacial, levando à ocorrência de todos os problemas mencionados para a cosmologia Newtoniana.

Assim, para conciliar a relatividade da inércia, uma distribuição de matéria que esteja em equilíbrio e que não implique num centro absoluto do universo e também,

\footnotetext{
$6 \longdiv { O }$ tensor de energia momento é uma quantidade conservada devido à invariância do sistema por quadri-translações. Suas componentes $T_{44}(x)$ e $T_{i 4}(x)$ representam energia e o vetor momento respectivamente sendo que $i=1, . ., 3$. Ele é a fonte de curvatura do espaço-tempo.

${ }^{7} \rho(x)$ designa a densidade média de matéria.
}

para que as velocidades dos corpos celestes estejam de acordo com as observações experimentais, Einstein postulou um universo espacialmente fechado, globalmente esférico. Nele as condições assintóticas não teriam cabimento e poderiam assumir um universo com seção espacial com densidade de matéria uniforme, e sem contradição com a relatividade da inércia, pois não haveria situação em que uma massa estaria totalmente afastada das demais. Apesar de seus interessantes esforços para conciliar seu modelo de cosmologia com a fundamentação conceitual Machiana, nomeadamente supor uma dada topologia ao espaço-tempo, no caso uma do tipo fechada, acabou-se por mostrar que seu modelo era instável 22]. Além disso, as observações cosmológias atuais favorecem a ideia de universo aproximadamente plano. Portanto, neste ponto e também naquele que se refere ao fato de que é possível eliminar localmente as influências das massas distantes através de uma transformação de coordenadas, nota-se uma certa divergência das predições de sua teoria com relação às ideias de E. Mach.

\section{A gravitação de Brans-Dicke}

Com o objetivo de conciliar a teoria da relatividade geral de Einstein com a totalidade das idéias de E. Mach, surgiu um conjunto de teorias gravitacionais alternativas. A mais conhecida delas é a de Carl.H Brans e R.H. Dicke (BD). Essa teoria parte do princípio da relatividade da inércia interpretando-a como dependente da disposição das massas no universo, o que está de acordo com as ideias de Mach. Em seu artigo 8 considera-se que tal efeito pode ser traduzido numa dependência da constante gravitacional $G$ com a posição e a distribuição de massas. Assim, esta constante seria entendida como um campo escalar.

A princípio poderia-se escolher outro escalar para representar o efeito das massas distantes, algo como uma estrutura formada por contrações dos objetos geométricos $\left(R, R_{\mu \nu}, \ldots\right)$ escalar de Ricci, tensor de Ricci, de Riemman, etc. Porém tais objetos decaem muito rapidamente com a distância e não poderiam descrever a influência das massas (ou melhor, massa-energia) distantes. Desse modo, ao considerar esse campo como o inverso da constante gravitacional tem-se, para baixas velocidades, a expressão aproximada:

$$
\phi(r) \sim \sum_{i} \frac{m_{i}}{r_{i} c^{2}}
$$

Tal solução apresenta um caráter harmônico e parece adequada a cumprir o papel mencionado, pois ela se propaga por todo o espaço ao invés de desvanecer. A interpretação seria a de uma interação gravitacional cuja intensidade dependeria da posição e da distribuição de matéria. De fato, na teoria de Brans-Dicke, verifica-se a relação: 


$$
\square \phi(r) \sim T
$$

Na equação acima tem-se o D'alembertiano generalizado para um sistema geral de coordenadas e $T$ representa o traço do tensor de energia momento $T=\eta^{\mu \nu} T_{\mu \nu}$ tomado com a métrica de Minkowski devido a aproximação para baixas velocidades:

$$
\eta_{\mu \nu}=\left(\begin{array}{cccc}
-1 & 0 & 0 & 0 \\
0 & -1 & 0 & 0 \\
0 & 0 & -1 & 0 \\
0 & 0 & 0 & 1
\end{array}\right)
$$

Logo, a distribuição de matéria afetaria a intensidade da interação gravitacional e definiria a maneira como ela varia no espaço-tempo.

Ainda com relação às perspectivas Machianas, também pode-se citar um comentário feito nesse artigo de que embora o princípio de equivalência fraco tenha uma forte base experimental, a sua versão forte, que, aparentemente viola a perspectiva Machiana, não a possui. Além disso, os autores ainda comentaram que a validade da geometria Riemmaniana na relatividade geral estaria, na visão deles, em ser uma possível e útil representação do fenômeno gravitacional, o que também é compatível com a visão estritamente fenomenológica de E.Mach.

\section{Comentários finais}

A teoria de Einstein elevou todos os sistemas de referência, inerciais ou não ao mesmo status não havendo sistemas privilegiados. As forças de inércia teriam realidade física, trataria-se da gravitação. Isto estaria de acordo com a réplica de Mach à questão de Newton. Desse modo, mover um sistema de modo não-uniforme com relação a outros possibilitaria gerar forças que, a priori, poderiam ser medidas. Com isto, Einstein implementou o relativismo, no qual as leis físicas devem se manter independente do referencial, e cujas possíveis forças de inércia não seriam uma falha mas um efeito físico incorporado pela teoria. De fato, isso não significa que Einstein adotou toda a abordagem Machiana haja vista que ela se referia à construção de uma física na qual todas as grandezas seriam referenciais e não haveria apelo a ideias metafísicas.

Daí um outro ponto importante. Como vimos, a teoria de Einstein faz uso do conceito de curvatura do espaçotempo e assume que essa é a causa das forças de inércia observadas entre referenciais com movimento relativo não uniforme. Essa curvatura não é observada diretamente mas apenas seus efeitos. O espaço-tempo que, de acordo com Mach, deveria ser meramente referencial ganha um conjunto de propriedades que não são observáveis. Um fato que embasa esta conclusão é a da construção da gravitação teleparalela 23 na qual se assume que o espaço-tempo tem torção, mas não curvatura, ao contrário da gravitação de Einstein, além do fato de que nela os efeitos inerciais podem ser mais facilmente separados daqueles puramente gravitacionais. Exatamente os mesmos resultados são obtidos a partir de ambas teorias. Pode-se mostrar que suas ações são iguais a menos de termos de fronteira. Além disso, a abordagem teleparalela permite separar eficientemente efeitos de coordenadas de efeitos puramente gravitacionais. Desse modo, fica a pergunta: O espaço-tempo dotado de propriedades geométricas sofisticadas seria apenas uma noção subjetiva de apoio como foi o éter para Maxwell?

Com relação aos princípios de equivalência fraco e forte, para que o conteúdo discutido no artigo possa ser entendido sem nenhuma ambiguidade, forneceremos uma definição mais precisa do que significa massa inercial e gravitacional dentro de um contexto relativista. No caso da gravitação universal tem-se que a massa, que é fonte do potencial gravitacional, e a massa inercial de um mesmo corpo são iguais. No caso relativista é mais apropriado trabalharmos em termos de energia-momento. No artigo [24] define-se a massa inercial como proporcional à energia canônica de repouso relacionada aos campos de matéria e de interação gravitacional. Esta energia é calculada por meio da soma das componentes 44 dos tensores de energia momentos canônicos dos campos mencionados. Basicamente pode-se mostrar que dentro de certas condições a fonte do campo gravitacional é equivalente ao tensor de energia momento canônico dos campos que a compõem, sempre que certas condições sejam respeitadas. Desse modo, por meio da estrutura da relatividade geral pode-se achar uma equação análoga à de Gauss para o eletromagnetismo $\partial_{k} E^{k} \sim \rho$, sendo que $E^{k}$ seria o campo elétrico e $\rho$ a densidade de carga. Já no caso gravitacional, a densidade seria de energia canônica dos campos, que está relacionada ao que se define como massa inercial. Pode-se mostrar que tal resultado pode é obtido por meio de uma integral de superfície numa região assintótica do espaço-tempo para a qual apenas a expansão de campo gravitacional fraco contribui. Assim, nessa situação o potencial gravitacional é proporcional à componente temporal deste campo fraco e pela sua forma pode-se fixar uma constante que faz o papel de massa gravitacional. $\mathrm{O}$ artigo mencionado demonstrou a igualdade dessas massas e, portanto, do princípio de equivalência fraco cuja verificação experimental através de versões modernas do experimento de Öetvos 17. mostrou que a razão entre tais massas é unitária com precisão de uma parte em $10^{13}$.

No caso de teorias gravitacionais alternativas, como a de Brans-Dicke, ocorre um acoplamento não-mínimo entre o campo escalar, que passa a representar a força da interação, e o campo que representa a ação da gravidade. Isso implica que os termos de fonte da gravitação não são mais equivalentes aos seus tensores de energia-momento canônicos e isso acaba por gerar uma massa inercial que é independente da gravitacional, violando o princípio de equivalência fraco. Além disso, o artigo discute como definir melhor e limitar o que seria o princípio de equiva- 
lência forte, comentando-se que sua base experimental é bem menos fundamentada do que a de sua versão fraca.

Para finalizar, é interessante citarmos que existem testes e limites experimentais para a observação de efeitos Machianos na natureza. Por exemplo, o mencionado referencial das estrelas fixas (que são os quasares e galáxias distantes.) não gira com relação ao nosso, ou seja constitui um bom referencial inercial, com a precisão de 0,00025 arcseg/ano. Como discutido no parágrafo anterior, existem resultados experimentais que limitam a validade do princípio de equivalência de Einstein, que dentre outras coisas, prevê que localmente podemos sempre assumir uma métrica Minkowskiana. Esse princípio parece se opor ao conceito Machiano de que até mesmo as distribuições de matéria distante são capazes de influenciar a física local. Assim, conhecer os limites de validade experimentais dos princípios de equivalência pode fornecer informações sobre qual a escala em que os efeitos Machianos poderiam começar a ser observados 25].

\section{Agradecimentos}

O autor, G. B. de Gracia, agradece à CAPES pelo suporte financeiro e ao B. M. Pimentel pela orientação e pelas importantes discussões sobre os fundamentos da física.

\section{Referências}

[1] J.J. Macionis e L.M. Gerber, Sociology (Seventh Canadian Edition, Pearson, 2010).

[2] A. Leite e S. Simon, Sci.Stud. 8, 2 (2010).

[3] E. Mach, The Science of Mechanics (The Open Court Publishing Co., Chicago, 1919).

[4] R.A. Martins, Rev. Bras. Ens. Fís. 2, 11 (2005).

[5] K.R. Popper, Conjecturas e Refutações (Editora UnB, Brasília, 1980).

[6] D.A. Mendonça, Os principia de Newton: Uma Leitura de seus Conceitos e Princípios Fundamentais (Uece, Iguatu, 2015).

[7] A.K.T. Assis, Mecânica relacional (CLE-Unicamp, Campinas, 1998).

[8] C. Brans e R.H. Dicke, Phys. Rev. D 124, 925 (1961).

[9] I. Newton, Principia. Princípios matemáticos da filosofia natural (Edusp, São Paulo, 2012), Livros I, II e III.

[10] E. Kant, Crítica da razão pura (Ed. Martin Claret, São Paulo, 2011).

[11] P. Davies, Inertia Theory - Paul Davies on the Meaning of Mach's Principle., disponível em http://www.padrak.com/ine/INERTIA.html.

[12] J. Bertrand, Os fundadores da astronomia moderna (Contraponto, Rio de Janeiro, 2003).

[13] I.B. Cohen, Isaac Newton's Papers and Letters on Natural Philosophy (Harvard University Press, Cambrige, 1978), $2^{\mathrm{a}}$ ed.

[14] H.C. Ohanian e R. Ruffini, Gravitation and space-time (Cambrige University Press, Cambridge, 2013), $3^{\mathrm{a}}$ ed.

[15] A. Einstein, Ann. d. Phys. 49, 146 (1916)
[16] R. Von Eötvös, in: Roland Eotvos Gesammelte Arbeiten, editado por P. Selenyi (Akademiai Kiado, Budapest, 1953).

[17] C.C. Speake e C.M. Will, Class. Quant. Grav. 29, 180301 (2012).

[18] E. Barausse, arXiv:1703.05699 (2017).

[19] V. Sabbata e M. Gasperini, Introduction to Gravitation (World Scientific Publishing Co, Singapore, 1985).

[20] A. Einstein, The principle of relativity (Dover, New York, 1952), p. 175.

[21] S.R.A. Salinas, Introdução à física estatística (Edusp, São Paulo, 1999), $2^{\text {a }}$ ed.

[22] D. Soares, Rev. Bras. Ens. Fís.34, 1 (2012).

[23] R. Aldrovandi e J.G. Pereira, Teleparallel Gravity, an introduction (Springer, Heidelberg, 2013), v. 1.

[24] H.C. Ohanian, arXiv:1010.5557 (2010).

[25] J. Barbour e H. Pfister, in: Mach's Principle; From Newton's Bucket to Quantum Gravity, edited by D. Howard e J. Stachel (Boston University, Boston, 1995), v. 6. 